\title{
Correlation of Structure with Crystalline to Amorphous Phase Transitions of 1,3,6-Substituted Fulvene Derived Molecular Glasses
}

Loren C. Brown, ${ }^{\mathrm{a}}$ Andrew J. Peloquin, ${ }^{\mathrm{a}}$ Nicholas P. Godman, ${ }^{\mathrm{b}}$ Gary J. Balaich, ${ }^{\mathrm{a}, *}$ Scott T. Iacono ${ }^{\mathrm{a}, *}$

aDepartment of Chemistry \& Chemistry Research Center, Laboratories for Advanced Materials, United States Air Force Academy, Colorado Springs, Colorado (USA)

bAir Force Research Laboratory, Materials and Manufacturing Directorate, Wright-Patterson Air Force Base, Dayton, Ohio (USA)

*Correspondence e-mail: gary.balaich@usafa.edu or scott.iacono@usafa.edu 


\section{Table of Contents}

\section{DESCRIPTION}

\section{PAGE NUMBER}

${ }^{1} \mathrm{H}$ and ${ }^{13} \mathrm{C}$ NMR spectra of 1,3-diphenyl-6-isopropylfulvene 2

${ }^{1} \mathrm{H}$ and ${ }^{13} \mathrm{C}$ NMR spectra of 1,3-diphenyl-6-cyclopropylfulvene 3

${ }^{1} \mathrm{H}$ and ${ }^{13} \mathrm{C}$ NMR spectra of 1,3-diphenyl-6-(2-thiophene)fulvene 5

S7-S8

${ }^{1} \mathrm{H}$ and ${ }^{13} \mathrm{C}$ NMR spectra of 1,3-diphenyl-6-(2,3-dimethoxynapthyl)fulvene 10

${ }^{1} \mathrm{H}$ and ${ }^{13} \mathrm{C}$ NMR spectra of 4-(cyclopentylmethyl)cyclopenta-1,3-diene-1,3-diyl)dibenzene 12b

Crystallographic Method

Table S1. Selected Bond Distances for Fulvenes 2, 8, 11, and cyclopentadiene 12b.

Table S2. Selected Bond Angles for Fulvenes 2, 8, 11, and cyclopentadiene 12b.

Table S3. Substituent Angles for Fulvenes 2, 8, 11, and cyclopentadiene 12b.

Table S4. Sample and crystal data for 1,3-diphenyl-6-isopropyl fulvene 2

Figure S1 and Figure S2. Thermal ellipsoid plot, molecular packing diagram, and $\pi$ - $\pi$ stacking for 2

Table S5. Sample and crystal data for 1,3-diphenyl-6-biphenyl fulvene 8

Figure S3. Thermal ellipsoid plot and molecular packing diagram for $\mathbf{8}$

Table S6. Sample and crystal data for 1,3-diphenyl-6-anthracene fulvene $\mathbf{1 1}$

Figure S4 and Figure S5. Thermal ellipsoid plot, molecular packing diagram, and $\pi-\pi$ stacking for 11 
1,3-diphenyl-6-isopropyl fulvene $2{ }^{1} \mathrm{H}-\mathrm{NMR}$ (500 MHz, $\mathrm{CDCl}_{3}$ )

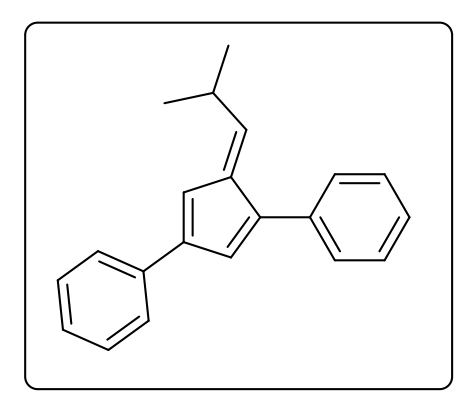

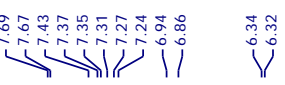
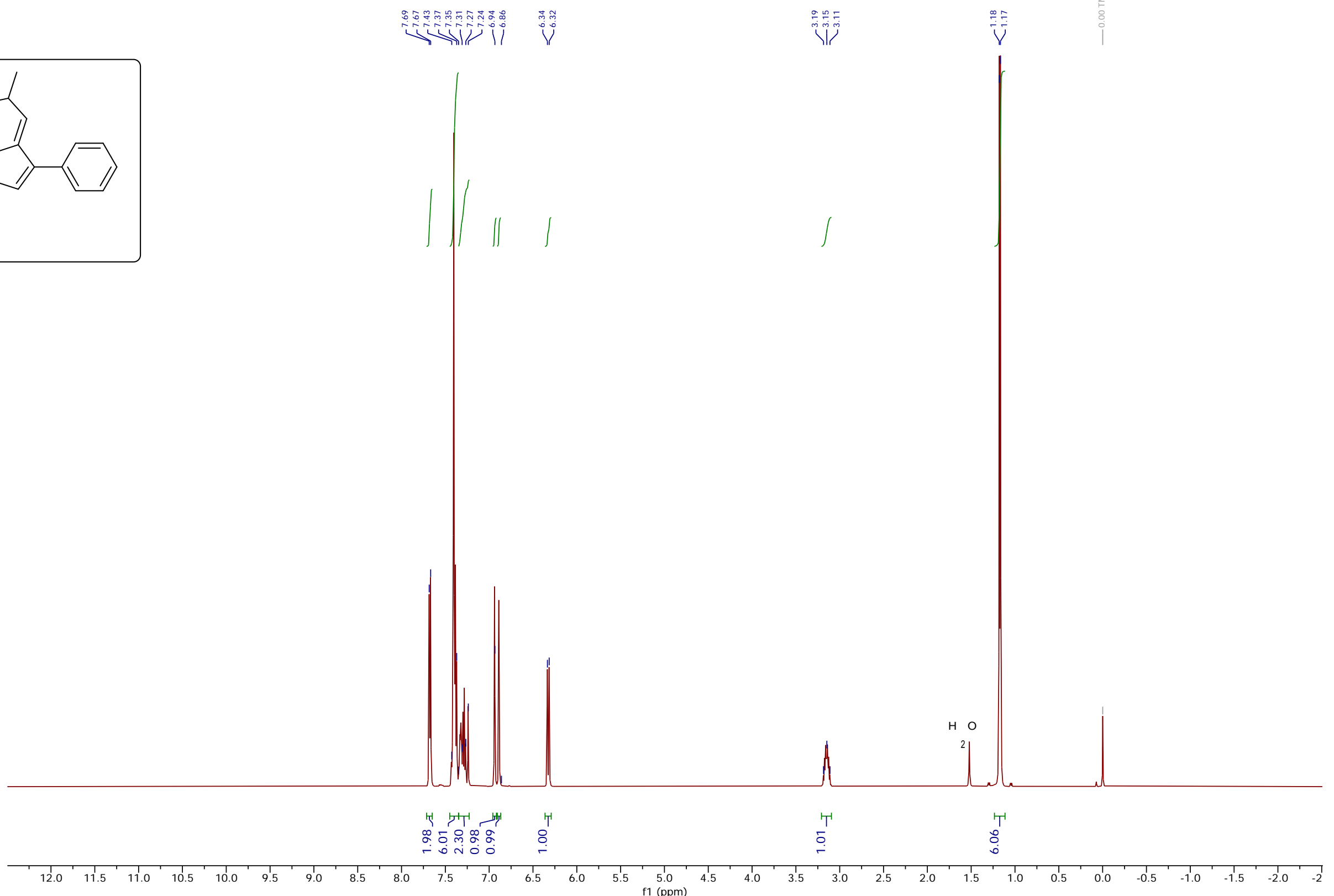
1,3-diphenyl-6-isopropyl fulvene $2{ }^{13} \mathrm{C}-\mathrm{NMR}$ (126 MHz, $\mathrm{CDCl}_{3}$ )



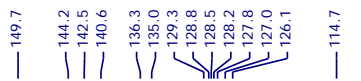




1,3-diphenyl-6-cyclopropyl fulvene $3{ }^{1} \mathrm{H}-\mathrm{NMR}$ (500 MHz, CDCl ${ }_{3}$ )
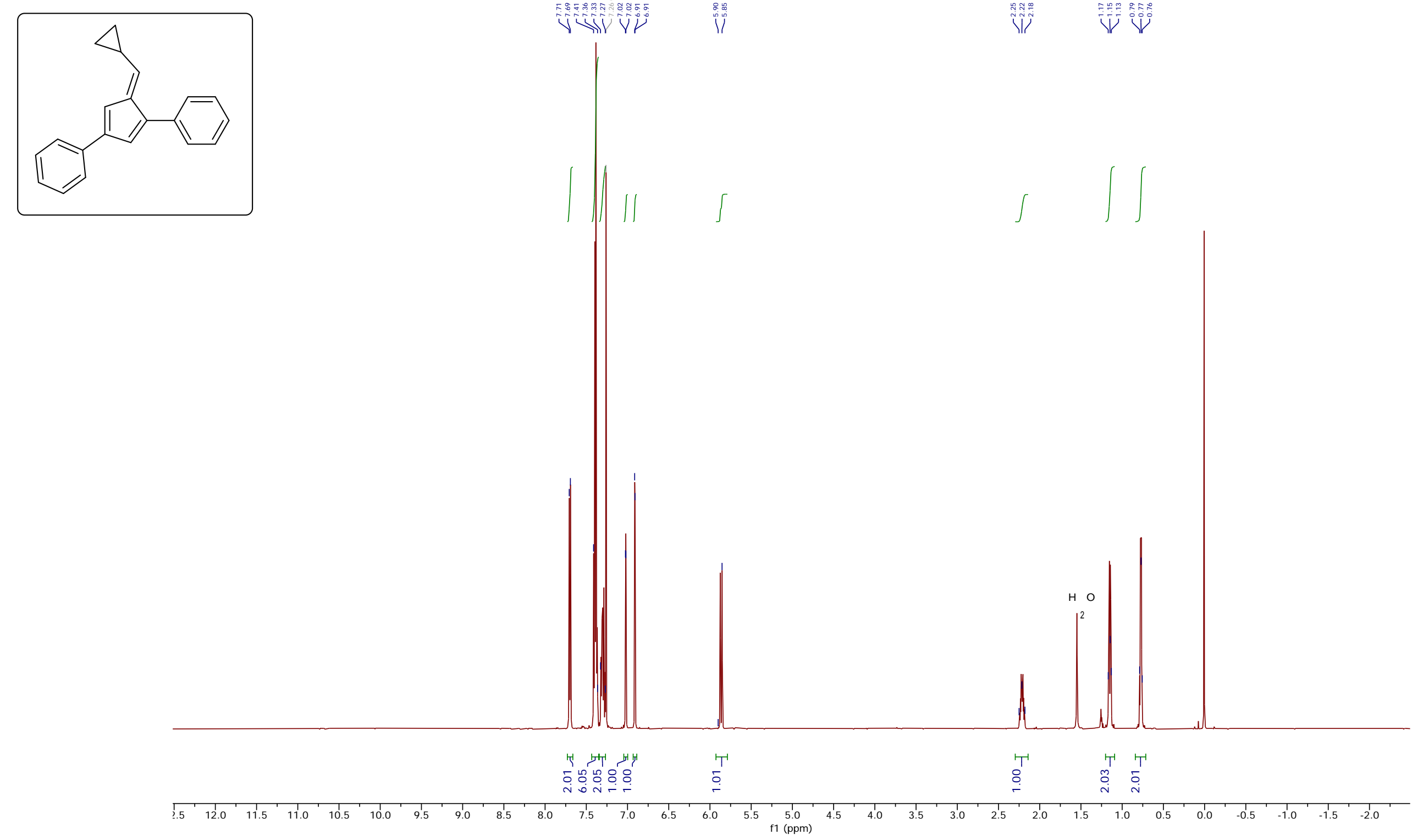
1,3-diphenyl-6-cyclopropyl fulvene $3{ }^{13} \mathrm{C}-\mathrm{NMR}\left(126 \mathrm{MHz}, \mathrm{CDCl}_{3}\right)$

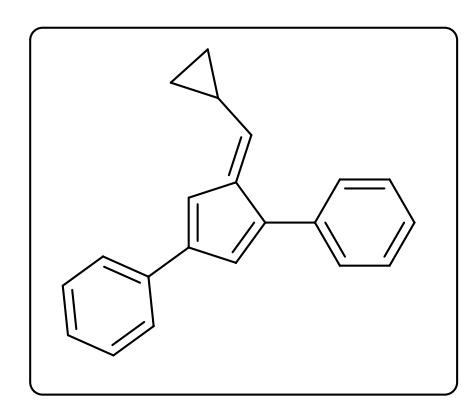



i i

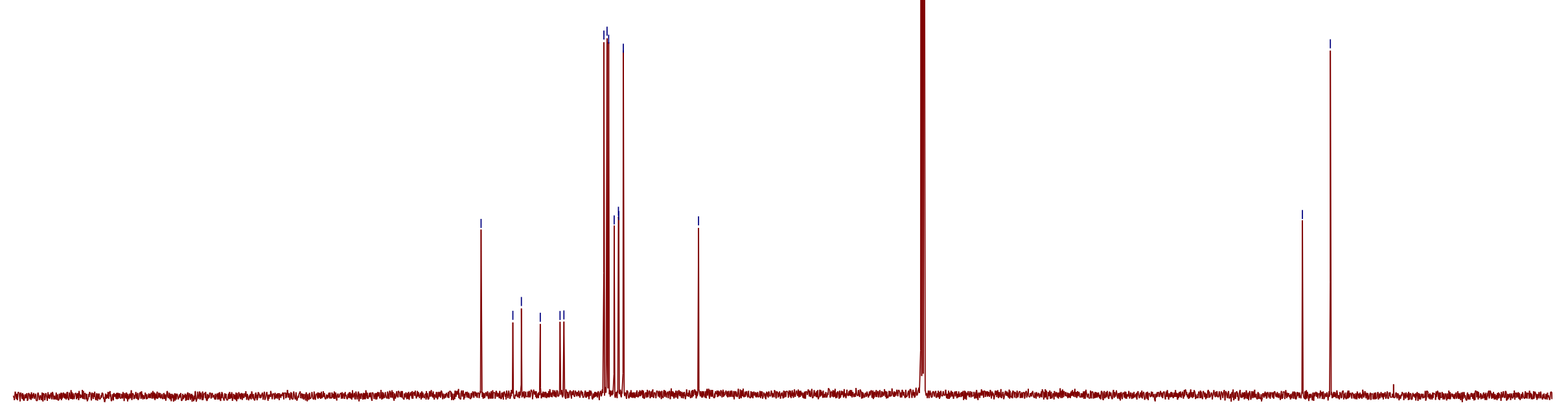


1,3-diphenyl-6-(2-thiophene) fulvene $5{ }^{1} \mathrm{H}-\mathrm{NMR}\left(500 \mathrm{MHz}, \mathrm{CDCl}_{3}\right.$ )

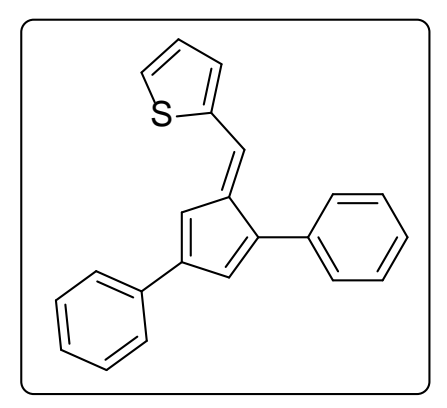

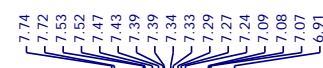

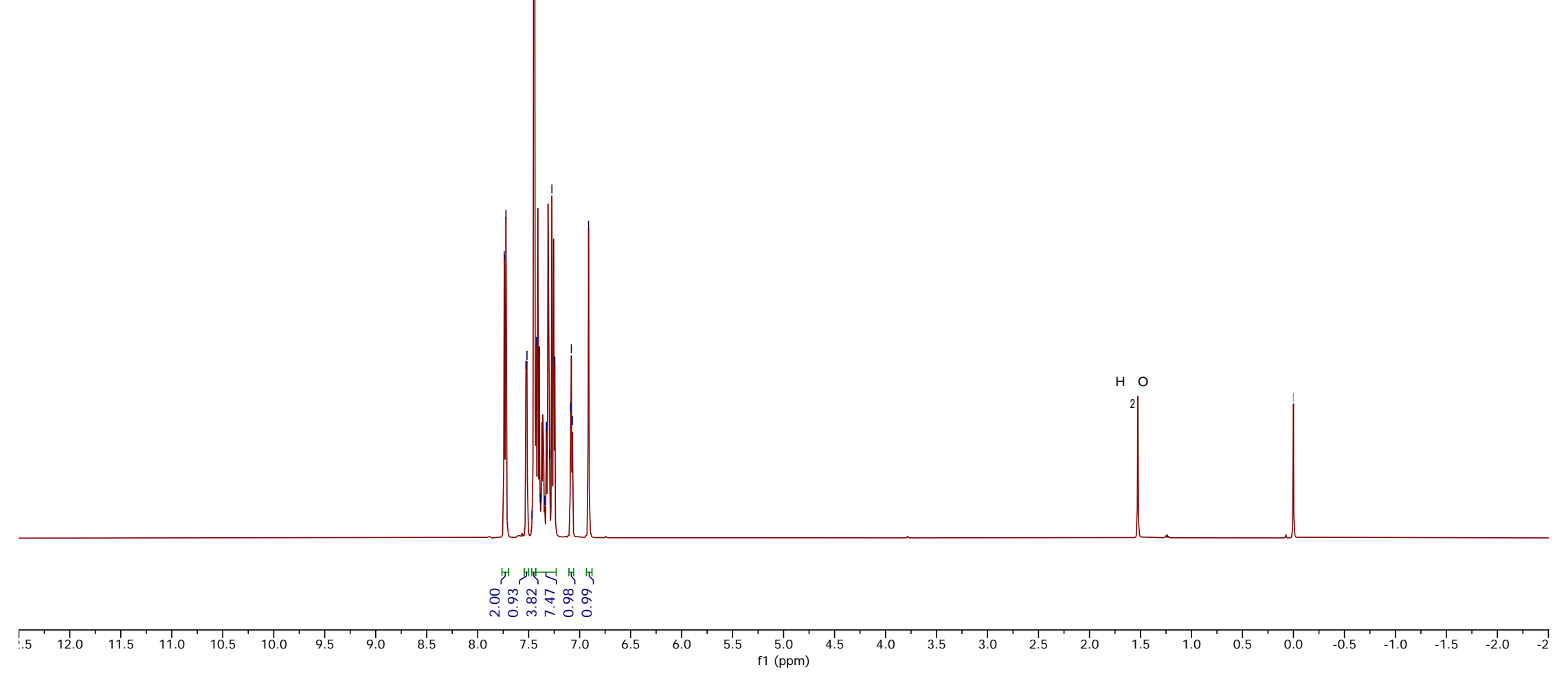


1,3-diphenyl-6-(2-thiophene) fulvene $5{ }^{13} \mathrm{C}-\mathrm{NMR}\left(126 \mathrm{MHz}, \mathrm{CDCl}_{3}\right.$ )

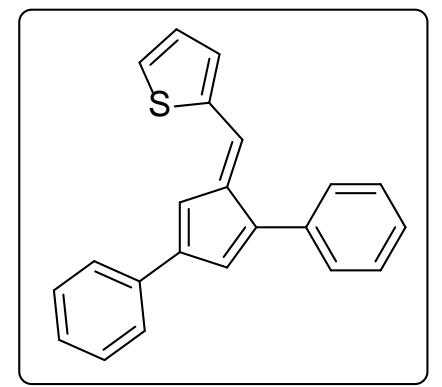



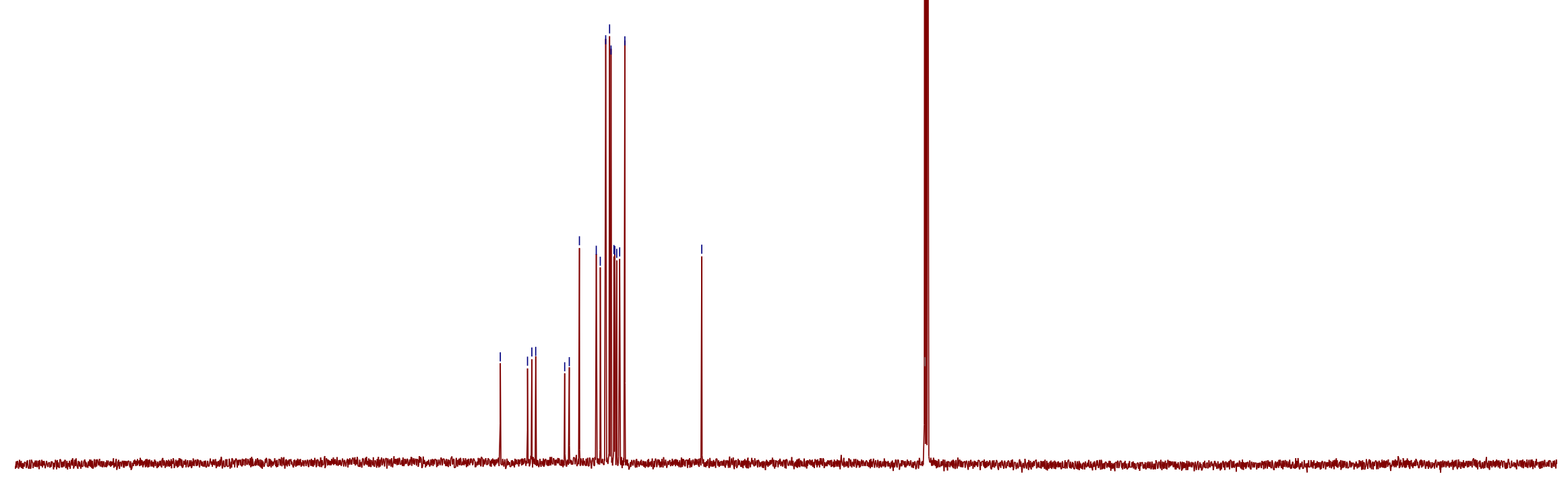




\section{1,3-diphenyl-6-(2,3-dimethoxynapthyl) fulvene $10{ }^{1} \mathrm{H}$-NMR}

(500 MHz, $\mathrm{CDCl}_{3}$ )
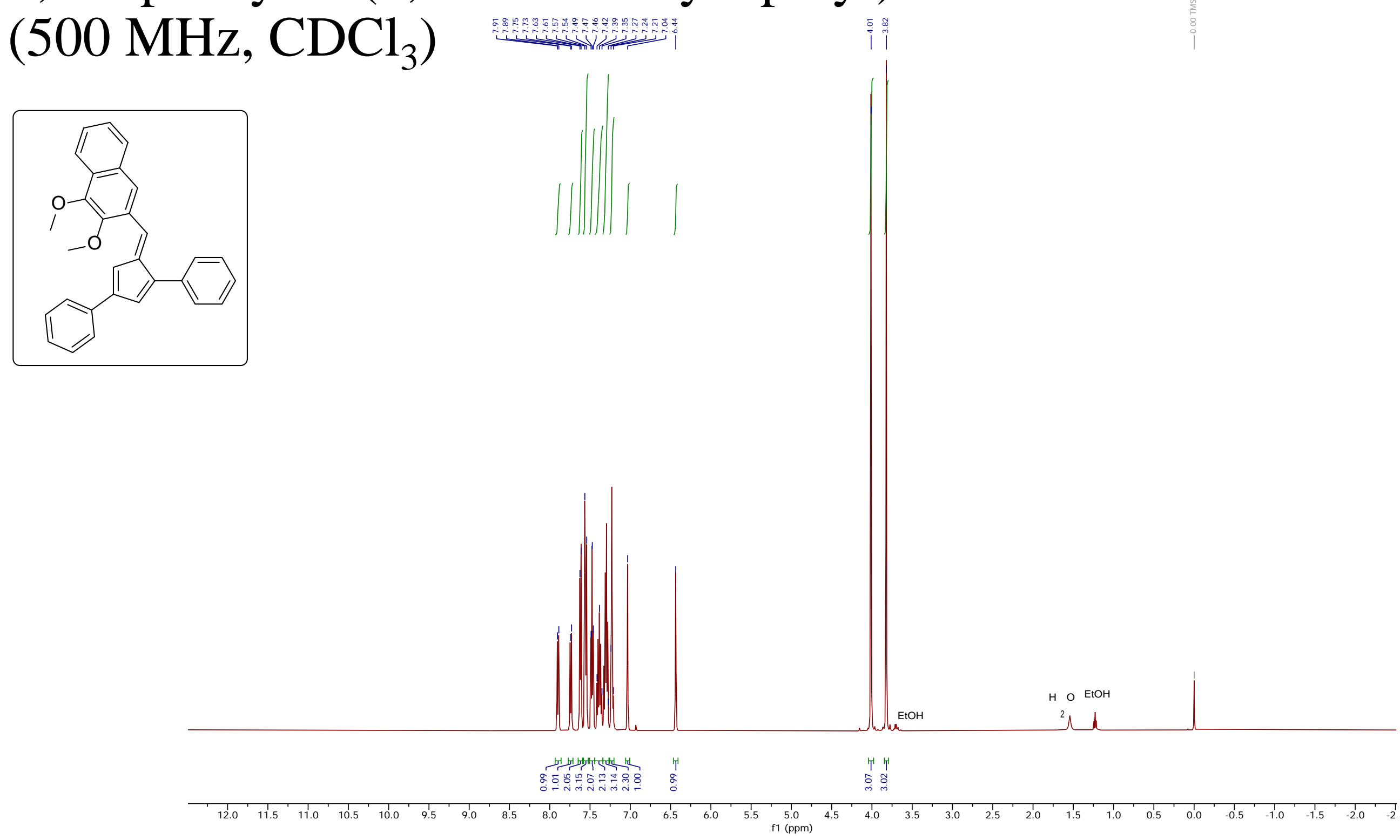
1,3-diphenyl-6-(2,3-dimethoxynapthyl) fulvene $1{ }^{13} \mathrm{C}$-NMR (126 $\left.\mathrm{MHz}, \mathrm{CDCl}_{3}\right)$

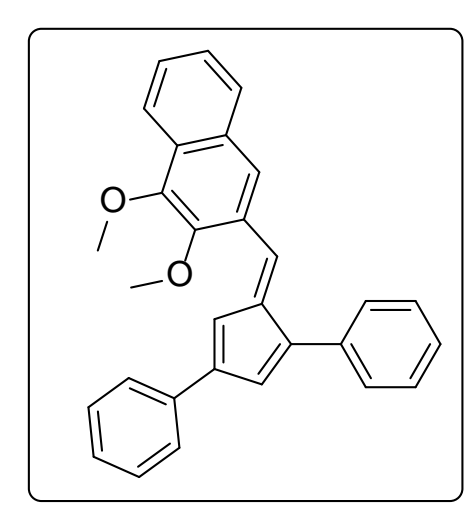

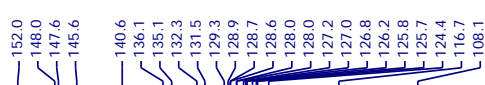

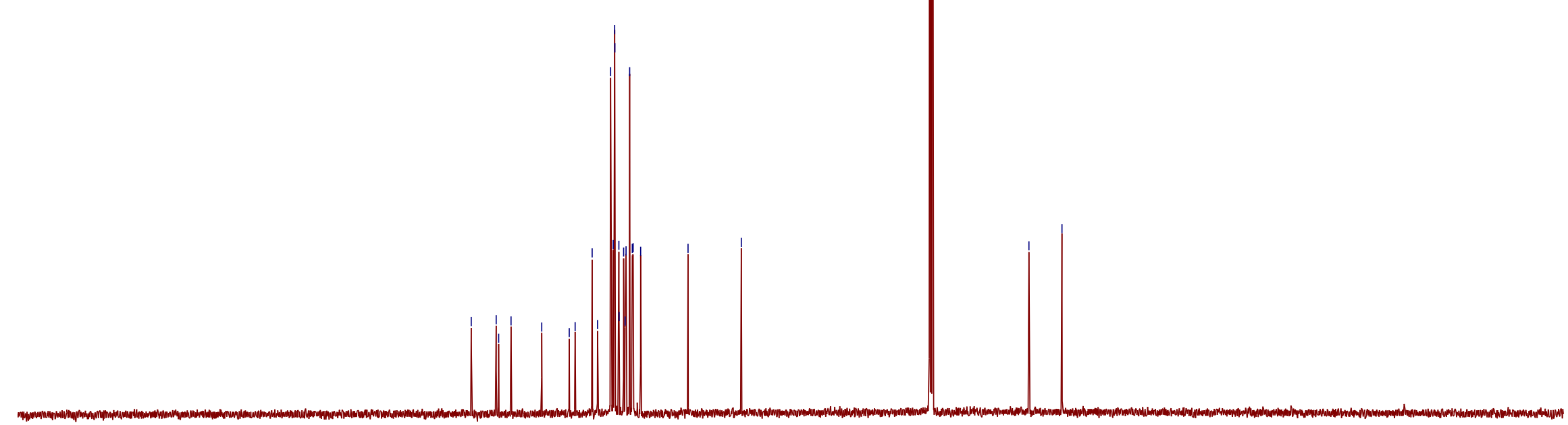


(4-(cyclopentylmethyl)cyclopenta-1,3-diene-1,3-diyl)dibenzene 12b ${ }^{1} \mathrm{H}-\mathrm{NMR}\left(500 \mathrm{MHz}, \mathrm{CDCl}_{3}\right.$ )

(4-(cyclopentylmethyl)cyclopenta-1,3-diene-1,3-diyl)dibenzene 12b ${ }^{13} \mathrm{C}-\mathrm{NMR}\left(126 \mathrm{MHz}, \mathrm{CDCl}_{3}\right)$

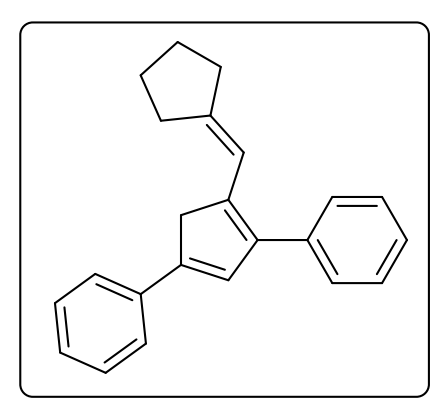


Crystallographic Details for Fulvenes 2, 8, 11, and cyclopentadiene 12b.

Crystals of fulvenes 2, 8, 11, and cyclopentadiene 12b were mounted on CryoLoops with Paratone oil, and data collected at $100 \mathrm{~K}$ on a Bruker SMART APEX II CCD diffractometer equipped with $\mathrm{Cu} \mathrm{K}_{\alpha}$ radiation $(\lambda=$ 1.54178). The data were integrated using the Bruker SAINT Software program and scaled using the SADABS software program. Solution by direct methods (SHELXT) produced a complete phasing model consistent with the proposed structure. All non-hydrogen atoms were refined anisotropically by full-matrix least-squares (SHELXL-2014). All carbon bonded hydrogen atoms were placed using a riding model. Their positions were constrained relative to their parent atom using the appropriate HFIX command in SHELXL-2014. Thermal ellipsoid views and packing diagrams were prepared using Olex2 or Mercury software. 
Table S1. Selected Bond Distances for Fulvenes 2, 8, 11, and cyclopentadiene 12b.

\begin{tabular}{|c|c|c|c|c|c|c|c|c|}
\hline \multirow{2}{*}{ Fulvene } & \multicolumn{4}{|c|}{ Ring Bond Distances $(\AA)$} & \multicolumn{3}{c|}{ Select Exocyclic Bond Distances $(\AA)$} \\
\cline { 2 - 9 } & C1-C2 & C2-C3 & C3-C4 & C4-C5 & C5-C6 & C1-C7 & C3-C13 & C6-C19 \\
\hline 6-Isopropyl 2 & $1.3531(17)$ & $1.4709(17)$ & $1.3608(17)$ & $1.4624(17)$ & $1.3461(17)$ & $1.4711(17)$ & $1.4730(17)$ & $1.4987(17)$ \\
\hline 6-Biphenyl 8 & $1.347(2)$ & $1.4705(19)$ & $1.359(2)$ & $1.465(2)$ & $1.354(2)$ & $1.4724(19)$ & $1.467(2)$ & $1.4685(19)$ \\
\hline 6-(Anthracene) 11 & $1.3523(14)$ & $1.4707(14)$ & $1.3593(15)$ & $1.4609(14)$ & $1.3445(14)$ & $1.4698(14)$ & $1.4681(14)$ & $1.4844(14)$ \\
\hline Cyclopentyl* 12b & $1.4558(14)$ & $1.3643(14)$ & $1.4914(14)$ & $1.5097(14)$ & $1.4667(15)$ & $1.4788(14)$ & $1.4649(14)$ & $1.3416(15)$ \\
\hline
\end{tabular}

Table S2. Selected Bond Angles for Fulvenes 2, 8, 11, and cyclopentadiene 12b.

\begin{tabular}{|c|c|c|c|c|c|c|c|}
\hline \multirow{2}{*}{ Fulvene } & \multicolumn{7}{|c|}{ Ring Bond Angles $\left(^{\circ}\right)$} \\
\cline { 2 - 8 } & C2-C1-C5 & C1-C2-C3 & C2-C3-C4 & C3-C4-C5 & C4-C5-C1 & C4-C5-C6 & C1-C5-C6 \\
\hline 6-Isopropyl 2 & $107.33(11)$ & $110.01(11)$ & $107.80(11)$ & $108.99(11)$ & $105.78(10)$ & $127.60(11)$ & $126.24(11)$ \\
\hline 6-Biphenyl 8 & $107.37(12)$ & $110.21(13)$ & $107.83(13)$ & $109.08(12)$ & $105.46(12)$ & $127.28(13)$ & $126.55(13)$ \\
\hline 6-(Anthracene) $\mathbf{1 1}$ & $107.06(9)$ & $110.05(9)$ & $108.03(9)$ & $108.78(9)$ & $106.01(8)$ & $126.20(9)$ & $127.68(9)$ \\
\hline Cyclopentyl* 12b & $109.12(9)$ & $110.13(9)$ & $108.17(9)$ & $104.60(8)$ & $107.97(9)$ & $124.97(9)$ & $126.83(10)$ \\
\hline
\end{tabular}


Table S3. Substituent Angles for Fulvenes 2, 8, 11, and cyclopentadiene 12b.

\begin{tabular}{|c|c|c|c|c|}
\hline \multirow{2}{*}{ Fulvene } & \multicolumn{4}{|c|}{ Substituent Bond Angles ( } \\
\cline { 2 - 5 } & 1-Ph & 3-Ph & 6-R & $\begin{array}{c}\text { Exo } \\
\text { bend }^{\mathrm{a}}\end{array}$ \\
\hline 6-Isopropyl 2 & $43.30(5)$ & $13.87(5)$ & $5.5(2)^{\mathrm{b}}$ & $13.6(2)$ \\
\hline 6-Biphenyl 8 & $30.34(6)$ & $20.14(7)$ & $46.67(6)$ & $12.7(3)$ \\
\hline 6-(Anthracene) 11 & $35.11(4)$ & $30.38(4)$ & $80.51(4)$ & $8.87(17)$ \\
\hline Cyclopentyl* 12b & $38.48(4)$ & $10.91(4)$ & $8.04(17)$ & $5.31(18)$ \\
\hline
\end{tabular}

${ }^{a}$ Measured with torsion angle, C7-C1-C5-C6

${ }^{b}$ Measured with torsion angle, C4-C5-C6-C19 


\section{Table S4. Sample and crystal data for 1,3-diphenyl-6-isopropyl fulvene 2}

Chemical formula

Formula weight

$\mathrm{C}_{21} \mathrm{H}_{20}$

272.37

Crystal system, space group

Monoclinic, $P 2_{1} / c$

Temperature (K)

100

$a, b, c(\AA)$

$\alpha, \boldsymbol{\beta}, \gamma\left({ }^{\circ}\right)$

$\mathrm{V}\left(\AA^{3}\right)$

$\mathbf{Z}$

Radiation type

$\mu\left(\mathrm{mm}^{-1}\right)$

Crystal size (mm)

Absorption correction

11.0596(2), 8.4681(1), 33.2511(5)

90, 99.015(1), 90

3075.62(8)

8

$\mathrm{Cu} \mathrm{K} \alpha$

0.49

$0.22 \times 0.2 \times 0.06$

Multi-scan

$\mathbf{T}_{\text {min }}, \mathbf{T}_{\text {max }}$

0.655, 0.754

No. of measured, independent and observed $[\mathrm{I}>2 \sigma(\mathrm{I})]$ reflections

$\mathbf{R}_{\text {int }}$

58081, 5589, 5261

$(\sin \theta / \lambda)_{\max }\left(\AA^{-1}\right)$

$R\left[F^{2}>2 \sigma\left(F^{2}\right)\right], w R\left(F^{2}\right), S$

0.033

0.602

0.038, 0.094, 1.08

5589

of reflections

384

H-atom treatment

H-atom parameters constrained

CCDC deposition \#

1988531 


\section{1,3-diphenyl-6-isopropyl fulvene 2}

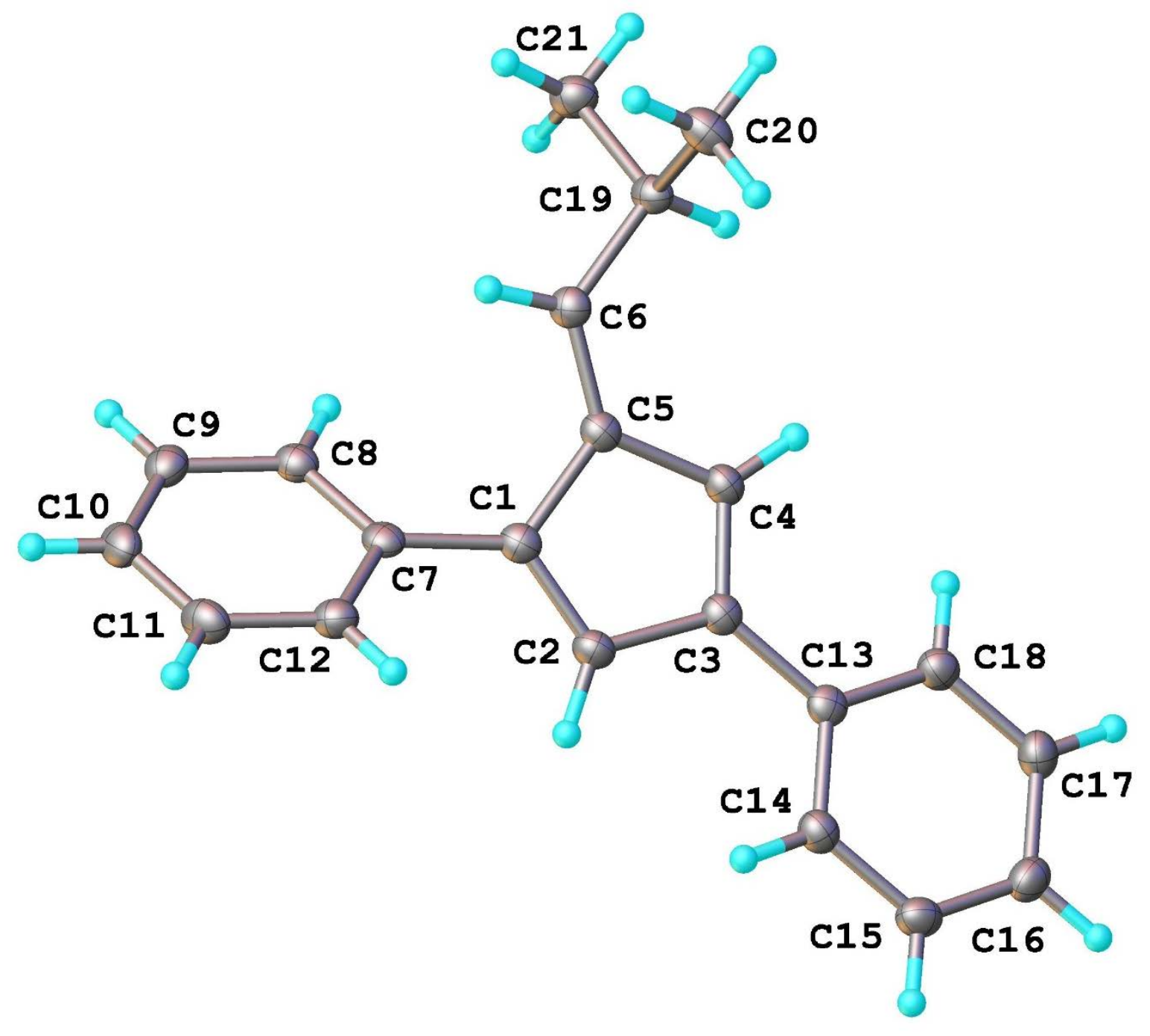

Figure S1. Thermal ellipsoid plot and molecular packing diagram (hydrogen atoms omitted for clarity) viewed down the a-axis for 2 (CSD: 1988531). Thermal ellipsoids shown at 50\% probability. 


\section{1,3-diphenyl-6-isopropyl fulvene 2}

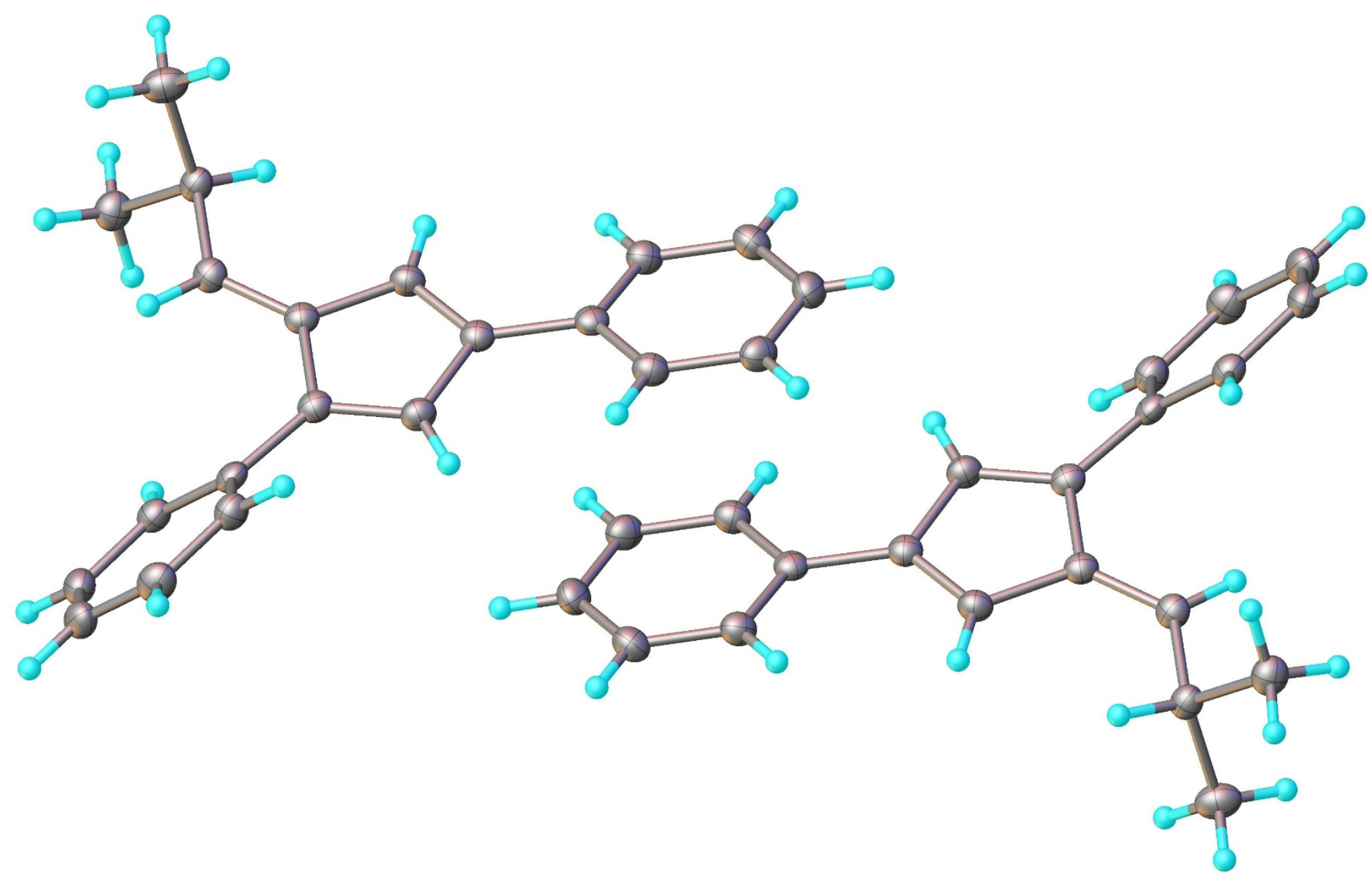

Figure S2. Example of $\pi-\pi$ interactions between neighboring 3-Ph moieties in the crystal structure of 2. Thermal ellipsoids shown at 50\% probability. The $\pi-\pi$ interacting 3 -Ph ring mean planes are separated by $3.377 \AA$. 
Table S5. Sample and crystal data for 1,3-diphenyl-6-biphenyl fulvene 8

\begin{tabular}{|c|c|}
\hline Chemical formula & $\mathrm{C}_{30} \mathrm{H}_{22}$ \\
\hline Formula weight & 382.47 \\
\hline Crystal system, space group & Monoclinic, $P 2_{1}$ \\
\hline Temperature (K) & 100 \\
\hline$a, b, c(\AA)$ & 11.7922(2), 7.3144(1), 23.5196(3) \\
\hline$\alpha, \boldsymbol{\beta}, \gamma\left({ }^{\circ}\right)$ & 90, 91.718(1), 90 \\
\hline$V\left(\AA^{3}\right)$ & 2027.72(5) \\
\hline $\mathbf{Z}$ & 4 \\
\hline Radiation type & $\mathrm{Cu} \mathrm{K \alpha}$ \\
\hline$\mu\left(\mathbf{m m}^{-1}\right)$ & 0.54 \\
\hline Crystal size (mm) & $0.15 \times 0.12 \times 0.06$ \\
\hline Absorption correction & Multi-scan \\
\hline $\mathbf{T}_{\min }, \mathbf{T}_{\max }$ & $0.680,0.753$ \\
\hline $\begin{array}{l}\text { No. of measured, independent and } \\
\text { observed }[I>2 \sigma(I)] \text { reflections }\end{array}$ & 48662, 7551, 7507 \\
\hline $\mathbf{R}_{\text {int }}$ & 0.023 \\
\hline$(\sin \theta / \lambda)_{\max }\left(\AA^{-1}\right)$ & 0.614 \\
\hline$R\left[F^{2}>2 \sigma\left(F^{2}\right)\right], w R\left(F^{2}\right), S$ & $0.026,0.069,1.06$ \\
\hline No. of reflections & 7551 \\
\hline No. of parameters & 542 \\
\hline H-atom treatment & H-atom parameters constrained \\
\hline CCDC deposition \# & 1988532 \\
\hline
\end{tabular}




\section{1,3-diphenyl-6-biphenyl fulvene 8}
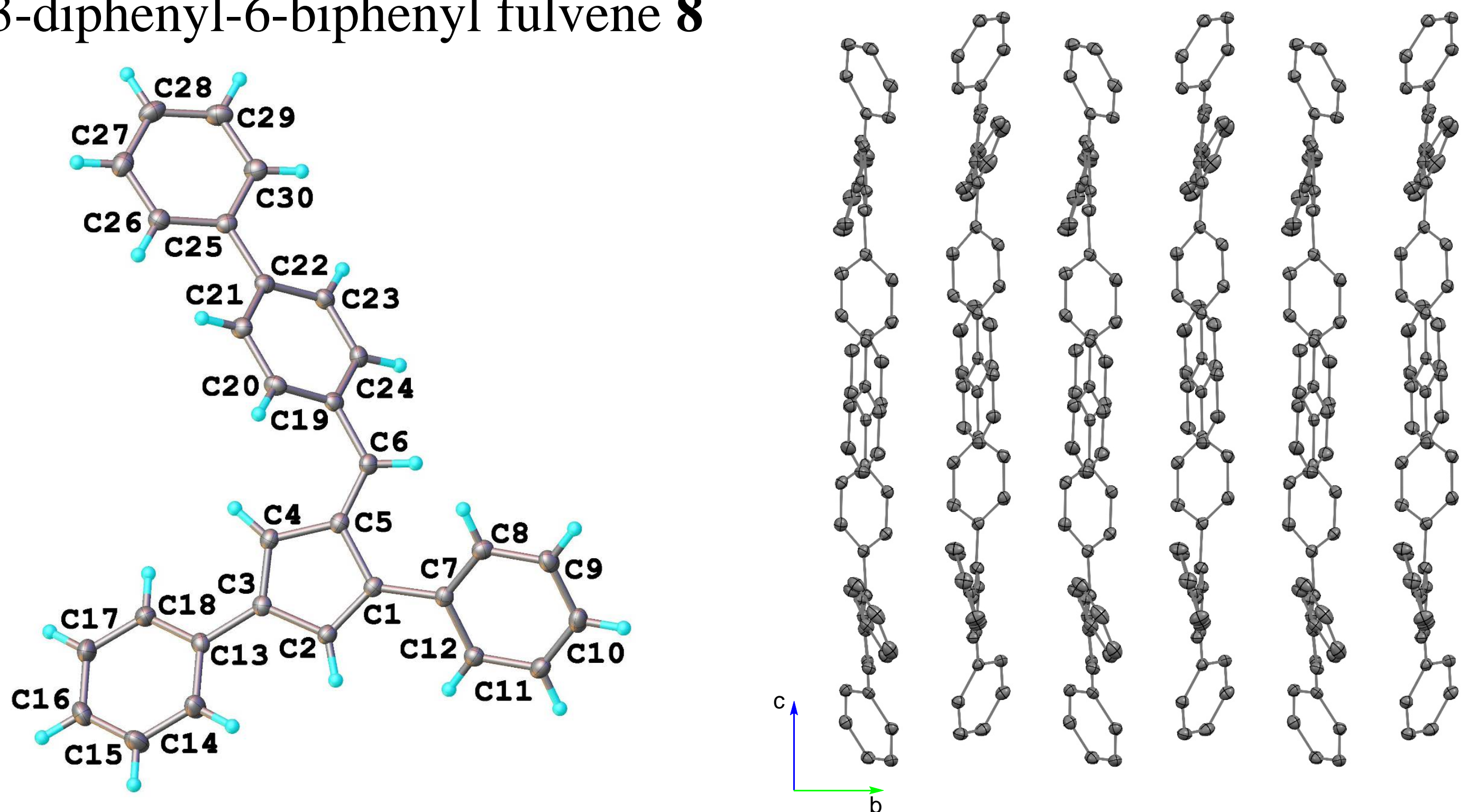

Figure S3. Thermal ellipsoid plot and molecular packing diagram (hydrogen atoms omitted for clarity) viewed down the a-axis for 8 (CSD: 1988532). Thermal ellipsoids shown at 50\% probability. 
Table S6. Sample and crystal data for 1,3-diphenyl-6-anthracene fulvene $\mathbf{1 1}$

\begin{tabular}{|c|c|}
\hline Chemical formula & $\mathrm{C}_{32} \mathrm{H}_{22}$ \\
\hline Formula weight & 406.49 \\
\hline Crystal system, space group & Monoclinic, $P 2_{1} / C$ \\
\hline Temperature (K) & 100 \\
\hline$a, b, c(\AA)$ & 9.4761(1), 9.6980(1), 23.3128(3) \\
\hline$\alpha, \beta, \gamma\left({ }^{\circ}\right)$ & $90,97.566(1), 90$ \\
\hline$V\left(\AA^{3}\right)$ & $2123.78(4)$ \\
\hline $\mathbf{Z}$ & 4 \\
\hline Radiation type & $\mathrm{Cu} \mathrm{K} \alpha$ \\
\hline$\mu\left(\mathbf{m m}^{-1}\right)$ & 0.55 \\
\hline Crystal size (mm) & $0.23 \times 0.12 \times 0.05$ \\
\hline Absorption correction & Multi-scan \\
\hline $\mathbf{T}_{\min }, \mathbf{T}_{\max }$ & $0.659,0.753$ \\
\hline $\begin{array}{l}\text { No. of measured, independent and } \\
\text { observed }[\mathrm{I}>2 \sigma(\mathrm{I})] \text { reflections }\end{array}$ & 29162, 4100, 3982 \\
\hline $\mathbf{R}_{\text {int }}$ & 0.024 \\
\hline$(\sin \theta / \lambda)_{\max }\left(\AA^{-1}\right)$ & 0.614 \\
\hline $\mathbf{R}\left[F^{2}>2 \sigma\left(F^{2}\right)\right], w R\left(F^{2}\right), S$ & $0.034,0.083,1.03$ \\
\hline No. of reflections & 4100 \\
\hline No. of parameters & 290 \\
\hline H-atom treatment & H-atom parameters constrained \\
\hline CCDC deposition \# & 1988529 \\
\hline
\end{tabular}




\section{1,3-diphenyl-6-(anthracene) fulvene 11}


Figure S4. Thermal ellipsoid plot and molecular packing diagram (hydrogen atoms omitted for clarity) viewed down the b-axis for 11 (CSD: 1988529). Thermal ellipsoids shown at 50\% probability. 


\section{1,3-diphenyl-6-(anthracene) fulvene 11}
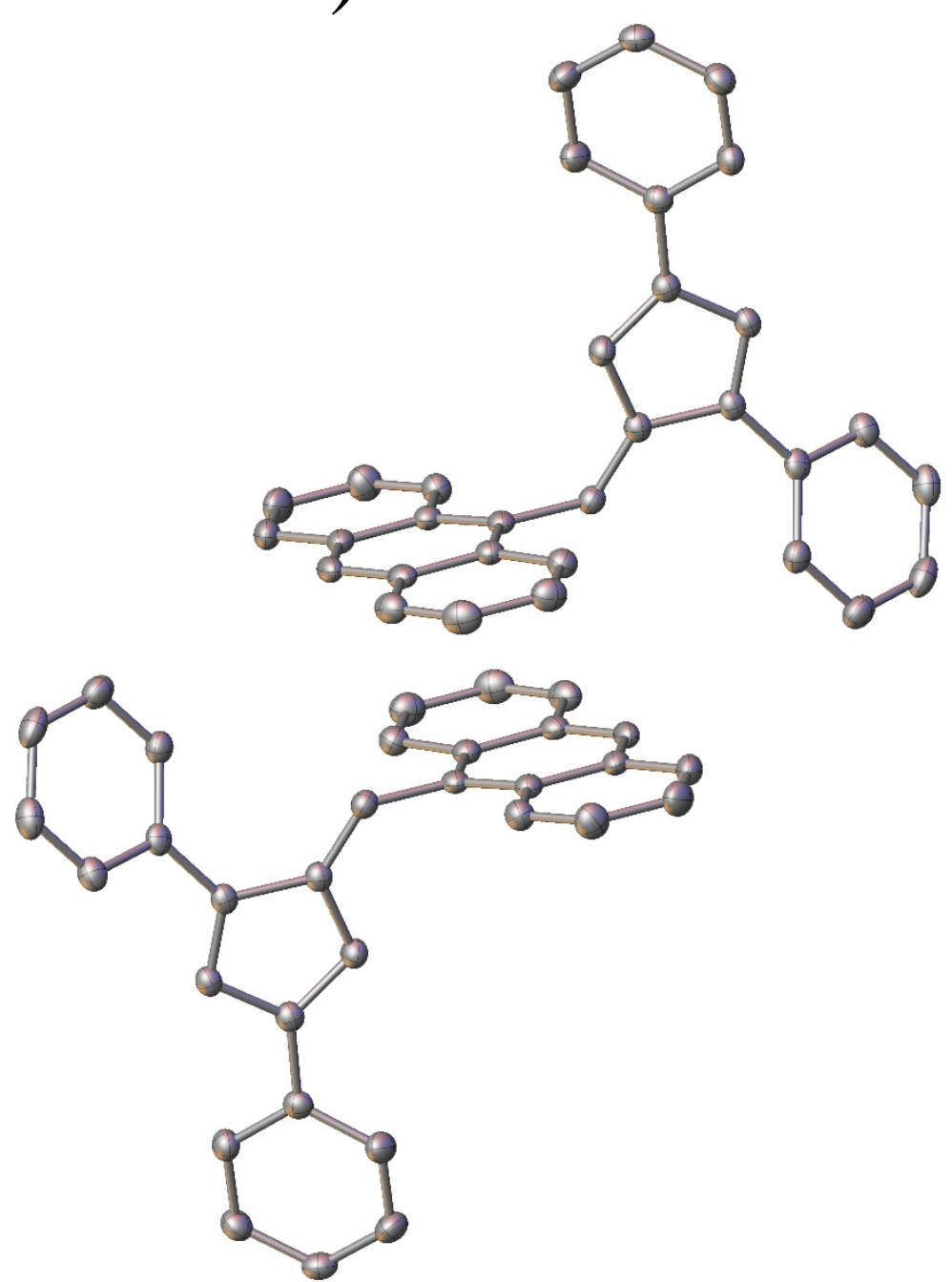

Figure S5. Example of $\pi-\pi$ interactions between neighboring 6-anthracene moieties. Thermal ellipsoids shown at $50 \%$ probability. Hydrogen atoms omitted for clarity. The $\pi-\pi$ interacting 6 -anthracene ring mean planes are separated by $3.491 \AA \AA$. 
Table S7. Sample and crystal data for (4-(cyclopentylmethyl)cyclopenta1,3-diene-1,3-diyl)dibenzene $\mathbf{1 2 b}$

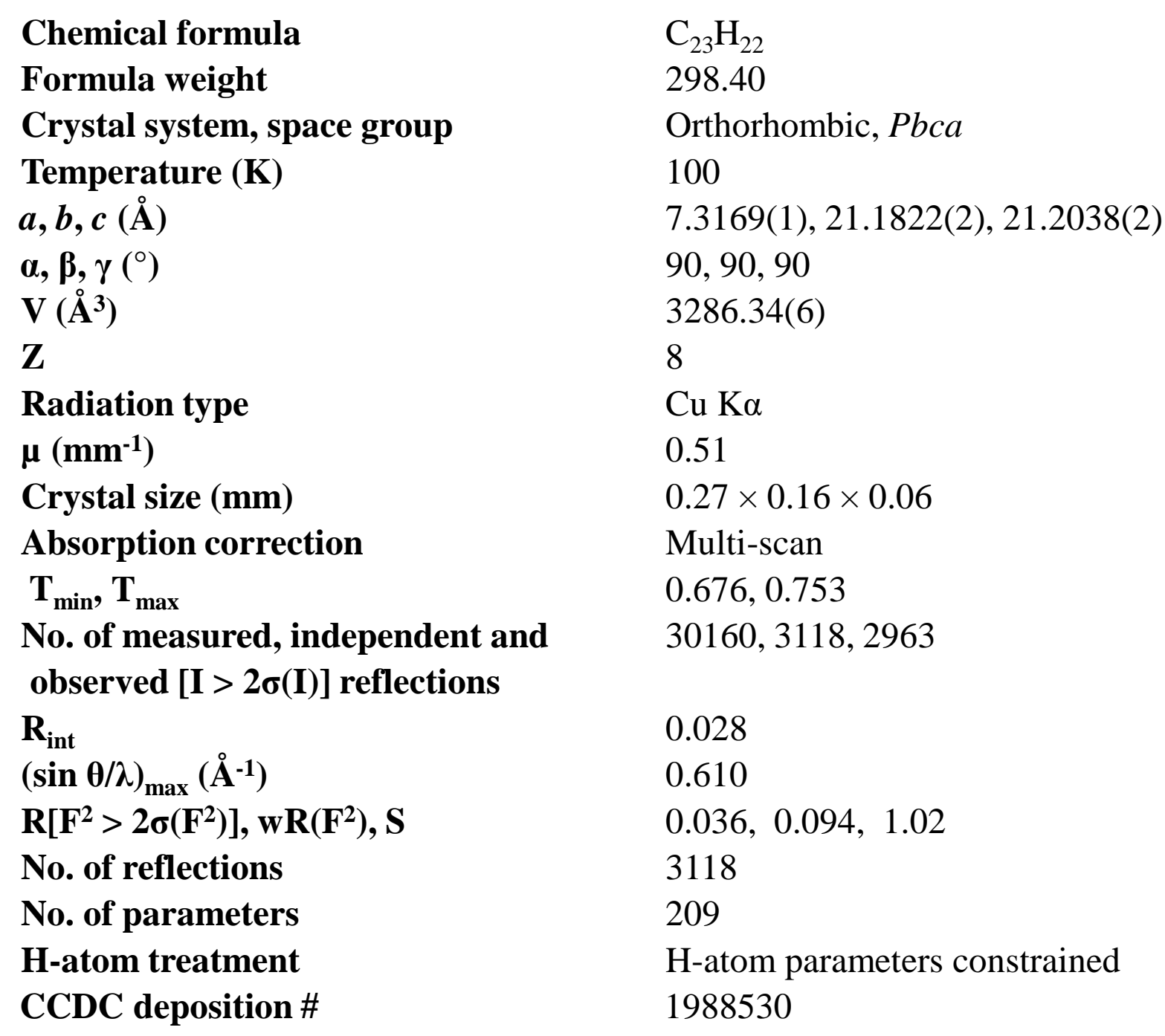


(4-(cyclopentylmethyl)cyclopenta-1,3-diene-1,3-diyl)dibenzene 12b
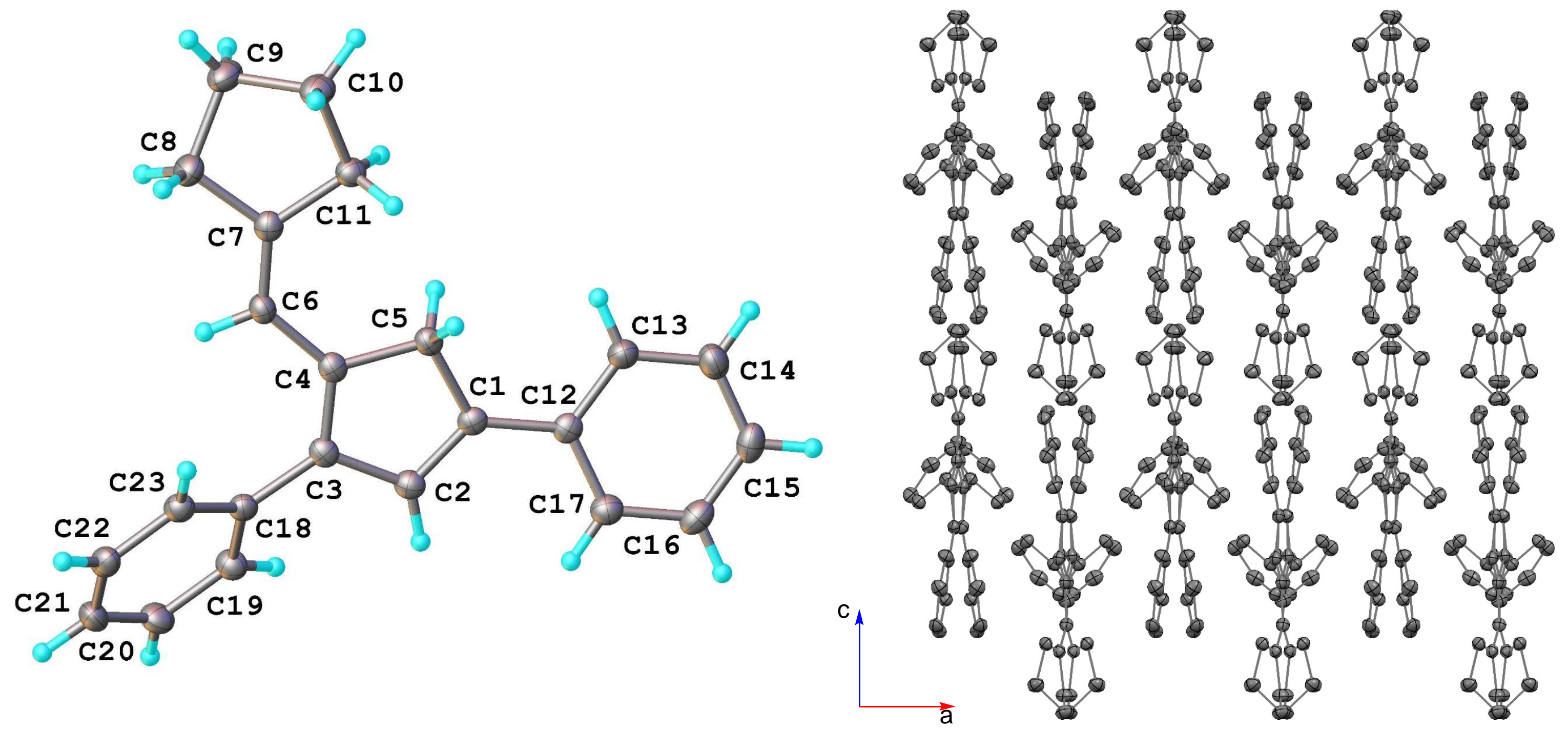

Figure S6. Thermal ellipsoid plot and molecular packing diagram (hydrogen atoms omitted for clarity) viewed down the b-axis for 12b (CSD: 1988530). Thermal ellipsoids shown at 50\% probability. 
9-(3,5-Dimethylbenzylidene)-8-(3,5-dimethylphenyl)-1,3,5,7tetraphenyl-2,4,7,8-tetrahydro-4,7-methanoazulene 13b

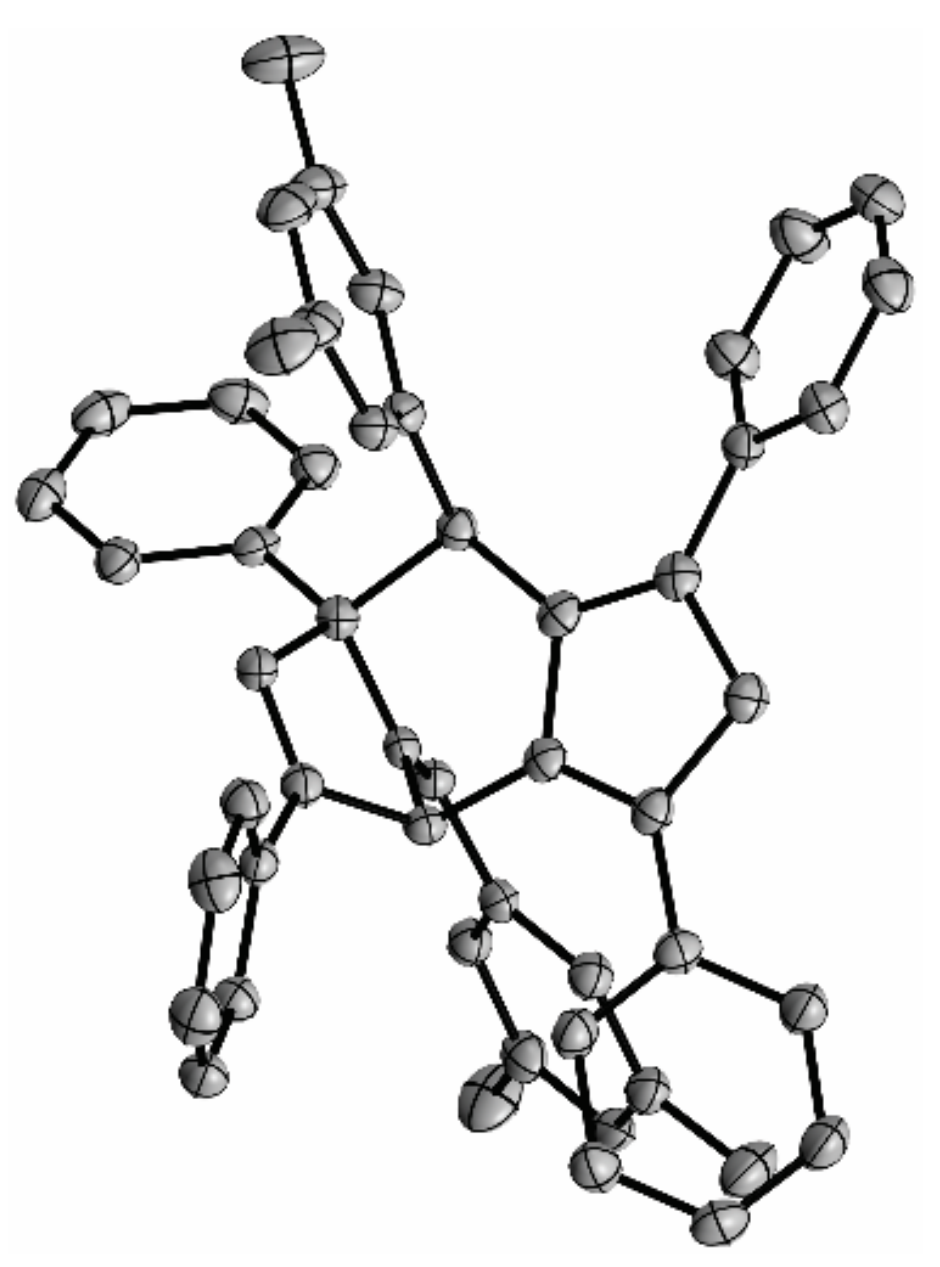

Figure S7. Thermal ellipsoid plot for 13b (CSD: 1835896). Thermal ellipsoids shown at 50\% probability. 